\title{
Correção cirúrgica da persistência do canal arterial em crianças de baixo peso e neonatos
}

\author{
Ciro Denevitz de Castro HERDY*, Fued Michel ABÍLIO*, Nelson VIEIRA*, \\ Sérgio Blanes BRANCAGLION ${ }^{*}$, Leonardo Silveira de CASTRO*, Júlio César Peclat de OLIVEIRA*, \\ Carlos Alberto M. PINTO*, Joăo B. THOMAZ*, Sérgio L. de AZEVEDO*
}

RBCCV 44205-306

\begin{abstract}
Herdy C D C, Abilio FM, Vieira N, Brancaglion B, Castro LS, Oliveira J CP. Pinto C A M, Thomaz J B, Azevedo $\mathrm{SL}$ - Correção cirúrgica da persistęncia do canal arterial em crianças de baixo peso e neonatos. ReV Bras Cir Cardiovasc 1996; 11 (3): 168-74.
\end{abstract}

RESUMO: A persistência do canal arterial ocorre com frequência em neonatos prematuros, provocando um grave quadro de disfunçăo cardiopulmonar. O tratamento envolve duas abordagens, sendo uma clínica e outra cirúrgica. A operação para a ligadura do canal arterial é praticada desde 1938 . O enfoque clínico preconiza o uso da indometacina, com o intuito de promover a oclusăo do canal arterial. O presente trabalho tem por objetivo avaliar os resultados obtidos com o tratamento cirúrgico da persistência do canal arterial, através de toracotomia eligadura em 14 pacientes, incluindo crianças de baixo peso e neonatos prematuros com quadro clínico instável. A principal indicaçăo cirúrgica, nestes casos, fol a presença de insuficiência respiratória aguda e insuficiência cardiaca. A técnica empregada foi a tripla ligadura do canal arterial. Nos 14 casos não obtivemos nenhum tipo de complicaçăo e sem mortalidade. A presença de uma Unidade de Tratamento Intensivo Neonatal (U.T.I) no Hospital foi de extremo valor no preparo dos pacientes e na evoluçăo no periodo de pós-operatório. Este trabalho comprova a eficácia do método cirúrgico empregado, com baixas taxas de morbidade e mortalidade e a importância da U.T.I neonatal no acompanhamento dos pacientes.

DESCRITORES: Persistência do conduto arterioso, cirurgia, prematuros neonatos. Prematuros neonatos, insuficiência respiratória, cirurgia. Prematuros neonatos, insuficiência cardiaca, cirurgia.

\section{INTRODUÇĀO}

Existe na atualidade um extenso debate sobre a escolha do tipo ideal de tratamento para a persistência do canal arterial ( $\mathrm{P} \mathrm{C} \mathrm{A),} \mathrm{principalmente}$ em crianças de baixo peso e neonatos prematuros, - que vem motivando diferentes pesquisas neste setor ${ }^{(1-3)}$. Galeno (131-201 DC), citado por SIEGAL (4), em 1962, em seus relatos sobre a circulação fetal, já havia observado a presença de um canal que comunicava a aorta à artéria pulmonar.
Em 1855, SKODA (5) e LANGER ${ }^{(6)}$, em 1857, descreveram pela primeira vez a histologia $e \quad 0$ mecanismo fisiológico do fechamento da P C A, após o nascimento.

GAYBRIEL et al. (7), em 1938, realizaram o primeiro fechamento cirúrgico da $\mathrm{P} C \mathrm{~A}$, em uma paciente portadora de endocardite, porém, sem efeito. No ano seguinte, GROSS \& HUBBARD (8) repetiram a mesma operação, com sucesso.

Em 1976, EDWARDS et al. (9) e FRIEDMAN et

Trabalho realizado no Hospital Universitário António Pedro. Universidade Federal Fluminense. Niteról, RJ, Brasil.

Apresentado ao $23^{\circ}$ Congresso Nacional de Cirurgia Cardiaca. Recite, PE, 20 a 23 de março, 1996.

* Do Hospital Universitário Antônio Pedro.

Endereço para correspondència: Ciro Denevitz de Castro Herdy. Rua Marquês de Paraná, 303. Niteról, AJ, Brasil. CEP; $24030-210$. Tel, (021) 719-2828. 
Herdy C D C, Abilio FM, Vieira N, Brancaglion B, Castro LS, Oliveira J CP, Pinto C A M, Thomaz JB, Azevedo SL - Correçāo cirúrgica da persistência do canal arterial em crianças de baixo peso e neonatos. Rev Bras Cir Cardiovasc 1996; 11 (3): $168-74$

al. (10) iniciaram o uso da indometacina, um inibidor da prostaglandina sintetase, para obter o fechamento da P C A.

MEIER et al. (11), em 1989 , realizaram vários trabalhos sobre o tratamento cirúrgico da P C A, em neonatos prematuros, indicando o seu fechamento nos casos de sindrome da angústia respiratória associada a insuficiência cardiaca.

A incidência da P C A nos pacientes prematuros é inversamente proporcional ao peso e à idade gestacional $(12,13)$. Em pacientes com menos de $1000 \mathrm{~g}$ $a$ incidència desta lesão ocorre entre $70 \%$ e $80 \%$. A P C A é responsável por $12 \%$ das cardiopatias congênitas, com predominância no sexo feminino na proporção de 2:1 (13).

WAY et al. (14), em 1979, relataram alteraçōes eletrocardiográficas sugestivas de isquemia subendocárdica em prematuros portadores de P C A e sindrome da angústia respiratória.

$\mathrm{Na}$ atualidade, tanto a ecocardiografia como o Doppler têm importância fundamental no diagnóstico deste tipo de má-formaçăo.

Năo existe unanimidade de opiniōes quanto ao método ideal para o fechamento da $P$ C $A$ e a melhora do estado funcional dos pulmőes. Diversas săo as opçōes, desde o tratamento clínico conservador, passando pelo uso de inibidores da prostaglandina sintetase, até a intervensāo cirúrgi$\mathrm{ca}(15,16)$. Em 1987, PALDER et al. (17), analisando 183 neonatos prematuros portadores de $\mathrm{P} \mathrm{C} \mathrm{A}$, observaram falha no tratamento com a indometacina em $42 \%$ dos casos sendo que, destes, $84 \%$ necessitaram da ligadura cirúrgica. A indicaçăo cirúrgica está baseada nos efeitos colaterais provocados pelo uso da indometacina, tais como hemorragia gastrintestinal e disfunção renal transitória $(10,18)$.

CALDERA \& BADOUAL (19), em 1981, propuseram o uso associado da vitamina $E$ e indometacina para o fechamento da P C A. HEYMANN et al. (20), em 1990, usaram a dexametasona associada ao tratamento convencional.

Existe uma grande controvérsia quanto ao tratamento cirúrgico. Alguns autores advogam a intervençăo cirúrgica somente nos casos sintomáticos. enquanto outros afirmam que a conduta de fechamento cirúrgico profilático reduz a morbidade em relaçăo ao tratamento farmacológico e clínico. BRANDT et al. (21) realizaram um estudo multicêntrico, verificando que os resultados cirúrgicos năo sofreram influência de idade, peso ao nascimento, doença pulmonar prévia ou uso de indometacina.

A mortalidade durante o ato cirúrgico é mínima e as complicações nāo sāo freqüentes, citando-se: sangramento, pneumotórax, reabertura do canal ar- terial, paralisia diafragmática, paralisia do nervo laríngeo recorrente e ligadura inadvertida da artéria pulmonar esquerda (21-23).

A mortalidade de crianças prematuras portadoras de $\mathrm{P} C \mathrm{~A}$ e não submetidas a intervenção cirúrgica chega, em alguns locais, à cifra de $100 \%$. Porém, mesmo sendo submetidas ao procedimento cirúrgico, existe a necessidade de uma unidade de tratamento intensivo neonatal para o preparo préoperatório e, fundamentalmente, para o acompanhamento no periodo de pós-operatório. Em nossa Intituição, antes da inauguração da Unidade de Tratamento Intensivo Neonatal (U.T.I. neonatal), a mortalidade era de quase $100 \%$. O presente trabalho vem mostrar que o tratamento cirúrgico da $\mathrm{P} \mathrm{C}$ A através da toracotomia e tripla-ligadura é eficaz e de baixa morbidade e mortalidade, desde que o hospital disponha de uma U.T.I neonatal para pré, per e pós-operatório destes pacientes e que a equipe seja altamente qualificada.

\section{CASUÍSTICA E MÉTODOS}

No Hospital Universitário Antônio Pedro, no período de 1983 a 1995 , foram submetidos à correção cirúrgica da P C A 14 pacientes, incluindo crianças de baixo peso e neonatos prematuros. Todos os pacientes foram internados na U.T.I neonatal do Serviço de Neonatologia e submetidos à rotina préoperatória, que incluía radiografia de tórax (PA), exames laboratoriais, como hemograma, coagulograma e rotina de bioquímica, eletrocardiograma e ecocardiograma. O tempo de internaçāo oscilou de 8 a 51 dias, com média de 20 dias. Dez $70 \%$ pacientes eram do sexo masculino, enquanto que 4 $(30 \%)$ eram do sexo feminino. A idade dos pacientes encontrava-se na faixa de $2 \mathrm{~m}$ a $6 \mathrm{~m}$, com média de 19 meses (Tabela 1). Somente 3 casos apresen-

TABELA 1

IDENTIFICAÇĀO DOS PACIENTES COM RELAÇÄO A IDADE EAO SEXO

\begin{tabular}{rllcc}
\hline CASO & PRONT. & NOME & SEXO & $\begin{array}{c}\text { IDADE } \\
\text { (MESES) }\end{array}$ \\
\hline 1 & 373.428 & RNM & M & 3 \\
2 & 328.499 & LAAF & M & 8 \\
3 & 371.746 & ROM & F & 32 \\
4 & 465.970 & HGM & F & 10 \\
5 & 445.598 & CSD & F & 7 \\
6 & 476.193 & IBS & M & 15 \\
7 & 449.388 & F/MNAA & M & 6 \\
\hline 8 & 474.570 & F/RCSO & M & 20 \\
9 & 478.099 & F/UN & M & 4 \\
10 & 476.517 & F/MA & M & 48 \\
11 & 477.511 & F/LFM & M & 16 \\
12 & 437.859 & F/AC & F & 21 \\
13 & 492.510 & WO & M & 60 \\
14 & 339.5566 & F/HNC & M & 68 \\
\hline & & & &
\end{tabular}


Herdy C D C, Abilio FM, Vieira N, Brancaglion B, Castro LS, Oliveira J CP. Pinto C A M, Thomaz J B, Azevedo S L-Correçáo cirúrgica da persistência do canal arterial em crianças de baixo peso e neonatos.

Rev Bras Cir Cardiovasc 1996; 11 (3):168-74.

GRÁFICO 1

PRINCIPAIS ANOMALIAS ASSOCIADAS A PCA

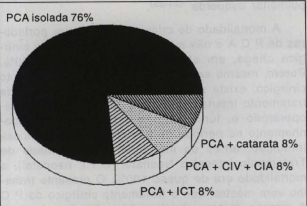

tavam lesōes associadas à P C A (Gráfico 1). O peso dos pacientes foi avaliado ao nascimento e no momento cirúrgico (Tabela 2). O uso de indometacina nāo foi preconizado na maioria dos pacientes, năo havendo, portanto, um protocolo quanto a indicaçăo de indometacina nesta casuística. A indicação cirúrgica foi principalmente baseada nos quadros de insuficiência respiratória, presente em $84,5 \%$, enquanto a pneumonia associada à insuficiência respiratória contribuiu com $15,5 \%$ dos casos.

Todos os pacientes foram operados seguindo rotina cirúrgica estabelecida pela equipe de cirurgia cardiovascular do Hospital Antônio Pedro. A sala de operação e os campos operatórios foram previamente aquecidos para evitar a perda de calor por parte dos pacientes. Os pacientes eram transferidos da isolete para uma mesa de operaçāo especial com sistema de aquecimento fotoelétrico. A monitorização nāo invasiva consistia na presença em sala de monitor cardiaco, oxímetro de pulso, termômetro

TABELA 2

PESO EM GRAMAS DOS PACIENTES

\begin{tabular}{ccc}
\hline CASO & PESO & $\begin{array}{c}\text { PESO } \\
\text { AONASCIMENTO }\end{array}$ \\
\hline NA CIRURGIA \\
\hline 1 & 3000 & 3450 \\
2 & 2300 & 3000 \\
3 & 3200 & 3450 \\
4 & 2800 & 3100 \\
5 & 2100 & 4100 \\
6 & 3100 & 3250 \\
7 & 2520 & 2600 \\
8 & 1050 & 1100 \\
9 & 3230 & 2800 \\
10 & 1530 & 2180 \\
11 & 2450 & 2600 \\
12 & 800 & 830 \\
13 & 2800 & 2860 \\
14 & 1600 & 1830 \\
\hline & & \\
\hline
\end{tabular}

retal, sonda vesical com saco coletor em circuito fechado e monitorização da pressăo arterial. $O$ estetoscópio foi fixado no precórdio do paciente para a ausculta dos batimentos cardiacos e da evoluçăo do sopro presente durante a operaçăo e para avaliação do desaparecimento, logo após o fechamento da P C A. O cateterismo venoso, principalmente em veia safena magna, $e$ a sonda nasogástrica foram também utilizados de rotina (Figura 1).

Após a induçăo anestésica, o paciente foi colocado em decúbito lateral direito, com coxim sob o tórax, com o braço esquerdo sobre a cabeça e fixado com fitas adesivas. A anti-sepsia da face látero-posterior do tronco e membros inferiores foi estabelecida como rotina.

A induçăo anestésica foi feita por via endovenosa, com Fentanil na dose de 10 micro-gotas $\mathrm{kg}$, seguido, quando necessário, da infusăo de Curare (Dibesilato de Atracurium), na dose de 0,08 a $0,1 \mathrm{mg} / \mathrm{kg}$.

A via de acesso à cavidade torácica definida como rotina pela equipe cirúrgica foi através de toracotomia lateral esquerda, com extensäo aproximada de $6 \mathrm{~cm}$, no $4^{2}$ espaço intercostal. Os músculos grande dorsal e redondo maior foram seccionados parcialmente. A abertura da cavidade torácica foi auxiliada por um afastador delicado do tipo "Finochetto", posto entre as costelas, O pulmāo foi afastado delicadamente com um afastador maleável envolvido em compressa úmida, para exposiçăo do hilo pulmonar e o canal arterial. A pleura mediastinal foi aberta anteriormente sobre a aorta, numa extensão de $2,5 \mathrm{~cm}$, junto à inserção do canal arterial, tendo a sua borda medial afastada através de fios de seda 2-0, como reparo.

O canal arterial era dissecado, evitando-se le. são de estruturas vasculares e do nervo laríngeo recorrente, isolado e reparado com fita cardiaca previamente umedecida. A técnica utilizada de rotina para o fechamento do canal arterial foi a tripla-

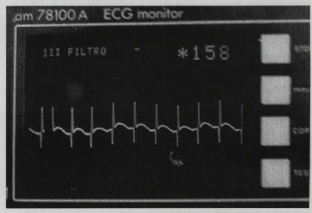

Fig. 1. Monitorizaçăo pré-operatớria, com auxilio de monitor cardiaco. 
Herdy C D C, Abilio FM, Vieira N, Brancaglion B, Castro LS, Oliveira J C P. Pinto C A M, Thomaz JB, Azevedo SL-Correçâo cirúrgica da persistência do canal arterial em crianças de baixo peso e neonatos.

ligadura. Esta técnica consiste na ligadura da extremidade abrtica e pulmonar do canal arterial com fios de Ethibond 2-0 $\theta$, associado a isto, confecção e fechamento em bolsa na extremidade aórtica com fio de Prolene 5-0. O controle cirúrgico imediato do fechamento eficaz do canal arterial foi realizado por meio da ausculta cardiaca, evidenciando-se o desaparecimento do sopro e do frêmito. Após a revisăo da hemostasia, foi realizada a sintese por planos com fio de Vicryl 3-0, e a pele fechada com sutura intradérmica com Nylon 6-0.

Todos os pacientes foram encaminhados a U.T.I neonatal, em berço aquecido, e permaneceram com a monitorizaçāo cardíaca e a oximetria de pulso no periodo de pós-operatório (Figuras 2 e 3 ). Toda a rotina de exames laboratoriais era novamente solicitada no período de pós-operatório imediato, incluindo gasometria arterial e radiografia de tórax. Todo este cuidado foi fundamental para o rápido desmame destes pacientes das próteses ventilatórias. Após o ato cirúrgico, os pacientes foram mantidos com um dreno de tórax, localizado na cavidade pleural esquerda, com um sistema de drenagem em selo d'água. Este dreno foi retirado no máximo após 24 horas, desde que nāo existisse drenagem importante. Os pacientes receberam alta em média com 10 dias de pós-operatório (Tabela 3), salvo os prematuros, que permaneciam no setor de pediatria para ganho ponderal. $O$ período de intubaçăo no pósoperatório oscilou entre um a dois dias (Tabela 4).

\section{COMENTÁRIOS}

Atualmente, o conceito sobre a persistência do canal arterial passou a ser mais complexo. Aceitase que o fechamento do canal ocorre em duas fases distintas: uma, onde o fechamento é funcional e se

TABELA 3

TEMPO MÉDIO DE INTERNACĀO HOSPITALAR NO PÓS OPERATORIO

\begin{tabular}{cc}
\hline CASO & POS-OPERATORIO (DIAS) \\
\hline 1 & \\
2 & 22 \\
3 & 6 \\
4 & 4 \\
5 & 2 \\
6 & 6 \\
7 & 9 \\
8 & 28 \\
9 & 4 \\
10 & 50 \\
11 & 45 \\
12 & 31 \\
13 & 12 \\
14 & 13 \\
\hline
\end{tabular}

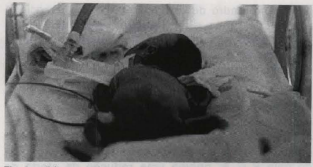

Fig. 2. Pós-operatório na U.T.I. neonatal em berço aquecido.

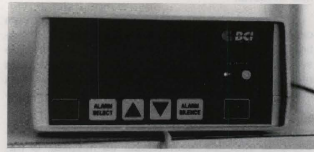

Fig. 3- Monitorizaçăo com oximetria de pulso no pós-operatório.

inicia logo após o nascimento $(10,13)$, e uma segunda fase, em que ocorre o fechamento anatômico, completando-se esta fase, no primeiro mês de vida, resultando o ligamento arterioso.

Na persistência do canal arterial ocorre um hiperfluxo pulmonar, levando os pacientes prematuros a um certo grau de disfunçăo cardíaca, que, somando-se ao quadro clínico de insuficiência respiratória, agrava ainda mais o quadro clínico. A falência cardiaca piora a função pulmonar, tornando-se, desta forma, importante cofator na decisão do momento cirúrgico. Nos casos relatados, todos os pacientes

TABELA 4

TEMPO DE INTUBAÇĀO ORO-TRAQUEAL (DIAS)

\begin{tabular}{rrrr}
\hline CASO & $\begin{array}{c}\text { TEMPONO } \\
\text { PRE-OPERAT. }\end{array}$ & $\begin{array}{c}\text { TEMPONO } \\
\text { POS-OPERAT. }\end{array}$ & TOTAL \\
\hline 1 & - & 1 & 1 \\
2 & - & 1 & 1 \\
3 & - & 1 & 1 \\
4 & - & 1 & 1 \\
5 & - & 1 & 1 \\
6 & - & 1 & 1 \\
7 & 6 & 2 & 8 \\
8 & 5 & 4 & 9 \\
9 & - & 1 & 1 \\
10 & - & 1 & 1 \\
11 & 16 & 28 & 44 \\
12 & 20 & 2 & 22 \\
13 & - & 1 & 1 \\
14 & - & 1 & 1 \\
\hline
\end{tabular}


Herdy C D C, Abilio FM, Vieira N, Brancaglion B, Castro LS, Oliveira J CP, Pinto C A M, Thomaz J B, Azevedo S L-Correção cirúrgica da persistência do canal arterial em crianças de baixo peso e neonatos. Rev Bras Cir Cardiovasc 1996; 11 (3):168-74.

possuiam quadro de disfunção pulmonar associado a diversos graus de disfunção ventricular. Estes dados foram criteriosamente avaliados mediante a comparaçăo dos achados radiográficos e ecocardiográficos (Figura 4).

As complicaçōes neurológicas săo bastante freqūentes, principalmente pelo fato do sistema circulatório encefálico ser de baixa resistência; a conseqũente diminuição do fluxo sangũineo durante a diástole, devido ao desvio aorto-pulmonar provocado pela $P$ C A, origina uma redução da oferta de oxigênio, ocasionando, portanto, quadros de isquemia cerebral.

Na casuística apresentada, nāo tivemos nenhum caso com complicaçōes neurológicas ou gastrintestinais, como a enterocolite necrotizante, quadro que se agrava em presença de síndrome da angústia respiratória; alguns relatos apontam a indometacina como fator coadjuvante para o aumento da incidência deste tipo de complicaçăo.

Todos os pacientes foram previamente avaliados pelo setor de neonatologia do hospital. O tratamento clínico inicial foi estabelecido segundo a literatura mundial, ou seja, através da restrição hídrica, aumento da $\mathrm{FiO}_{2}$, e uso de diuréticos como substâncias vasoativas, com o objetivo de diminuir a sobrecarga de volume. Além desta rotina, o uso da indometacina foi tentado em alguns casos, entretanto nāo se estipulou um protocolo desta substância, dificultando, portanto, a avaliação de sua eficácia. Acredita-se que o resultado năo satisfatório obtido com a indometacina é devido a rápida recuperação da produçāo de prostaglandinas, levando, então, ao relaxamento da musculatura do canal arterial.

O tratamento cirúrgico estipulado para estes pacientes teve uma modificação da técnica original, pois, além da ligadura proximal e distal, foi feita uma sutura em bolsa na extremidade aórtica com fio de Prolene 5-0. Esta técnica mostrou-se bastante eficaz, visto que nāo houve nenhum caso de reabertura do canal.

Apesar de alguns autores advogarem o acesso ao canal arterial por via extrapleural, nós preferimos a via transpleural, o que nāo motivou qualquer tipo de complicaçāo como o pneumotórax. Achamos que essa complicação está mais relacionada ao manuseio incorreto do dreno torácico.

A conduta quanto à indicaçăo cirúrgica na $\mathrm{P} \mathrm{C}$ A é controversa, uma vez que o canal pode fecharse espontaneamente com o crescimento. Entretanto, sabe-se que o fechamento precoce da P C A, principalmente em prematuros, diminui a necessidade de ventilação mecânica e reduz as complicaçōes, como displasia broncopulmonar, enterocolite

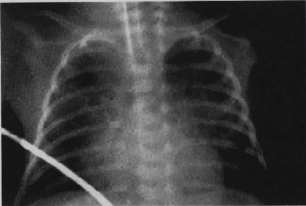

Fig. 4. Radiograflia de tórax em P. A, evidenciando o aumento da área cardíaca.

necrotizante e intolerância às alimentaçōes entéricas. O tratamento com a indometacina mostra-se, segundo a literatura, tão eficaz quanto a cirurgia, principalmente nos neonatos de baixo peso ao nascimento (menos de $1000 \mathrm{~g})(10-13)$, embora com um indice de insucesso de $42 \%$ (12).

A reabertura do canal arterial após a operaçăo năo ocorre com freqüencia. Alguns autores relatam a sua experiência com clipes metálicos como sendo também eficaz; entretanto este tipo de técnica está sujeita a algumas limitaçōes.

Um dos fatores principais para os bons resultados cirúrgicos é a disposiçāo de uma infra-estrutura capaz de fornecer o suporte adequado aos pacientes, tanto no pré, quanto no pós-operatório. Neste ponto, a U.T.I neonatal do Hospital Antōnio Pedro mostrou-se extremamente eficaz, dotada de equipamentos e pessoal treinado. A U.T.I neonatal precisa dispor de ventiladores mecânicos de última geração para facilitar o rápido desmame das próteses ventilatórias no pós-operatório (Figura 5).

A evolução pré-operatória é de fundamental importância para o bom resultado da operaçăo. Nos

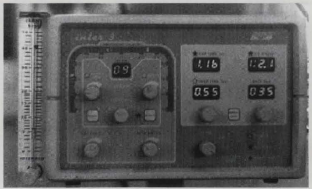

Fig. 5 - Ventilador mecănico microprocessado utilizado no pré e pos-operatorio. 
casos aqui apresentados, todos os pacientes passaram por uma criteriosa avaliaçăo clínica, laboratorial e de imagem. Nas radiografias de tórax, os principais achados foram a congestão pulmonar $e$ - aumento da área cardiaca. O exame ecocardiográfico foi realizado em todos os pacientes, fornecendo importantes dados, como as dimensōes do canal arterial e a presença de mal-formaçőes associadas.

No exame eletrocardiográfico, podemos observar alteraçōes da onda $T$ (inversăo de $T$ ) e desvio do eixo para a direita. Estas alteraçōes, entretanto, sảo inespecíficas, podendo ocorrer em crianças de baixo peso ou neonatos prematuros sem P C A.

Năo houve mortalidade na presente série. Atribuimos esse ótimo resultado, principalmente ao acompanhamento rigoroso por parte da equipe da U.T.I. neonatal. As complicaçōes também năo foram relevantes, estando relacionadas apenas ao maior tempo de permanência das próteses ventilatórias em 2 pacientes.

Apesar do advento da toracoscopia para tratamento da P C A, este tipo de técnica mostra um elevado grau de dificuldade em crianças de baixo peso e, principalmente, nos neonatos prematuros em condiçōes clínicas precárias. Em nossa expe- riência, portanto, a toracotomia a céu aberto, apesar do elevado grau de agressão, mostra-se eficaz e com vantagens de acesso nos casos de complicaçōes como a rotura do canal antes de sua ligadura.

\section{CONCLUSÕES}

A U.T.I. neonatai, associada a uma equipe altamente especializada e treinada, é de fundamental importância para o sucesso destes pacientes, principalmente no preparo pré, per e pós-operatório.

O tratamento cirúrgico, associado à instalação de uma U.T.I. neonatal no Hospital Universitário Antônio Pedro, mudou o prognóstico de $100 \%$ de mortalidade anteriormente para $0 \%$ nos pacientes enviados para o tratamento cirúrgico.

Nāo obtivemos complicaçöes operatórias.

O acesso transpleural não apresentou dificuldade técnica, mesmo nos pacientes de baixo peso.

A tripla-ligadura mostrou ser uma técnica eficaz para o fechamento da $\mathrm{P} C \mathrm{~A}$, năo sendo constatado nenhum caso de reabertura do canal. Todos os pacientes obtiveram melhora do quadro clínico de insuficiência respiratória $e$ cardiaca.

Herdy CD C, Abilio FM, Vieira N, Brancaglion B, Castro LS, Oliveira J C P. Pinto C A M, Thomaz JB, Azevedo $\mathrm{SL}$. Surgical treatment of patent ductus arteriosus in low-weight child and neonates. Rev Bras Cir Cardiovasc 1996; 11 (3): 168-74.

ABSTRACT: The patent ductus arterious frequently occurs in premature neonates causing serious cardiopulmonary disfunction. The treatment includes two options; one clinic and the other surgical procedure. The surgery for patent ductus arteriosus has been done since 1938. Clinical treatment with indometacin induces closure of the arteriosus ductus. The aim of the article is to analize the surgical results with thoractomy by triple-ligature of the ductus for the treatment of persistent ductus arteriosus in fourteen patients including low weight children and premature neonates with cardiopulmonary disfunction. The indications for surgery in these cases were respiratory distress and congestive heart failure. The technique used was triple-ligature of the patent ductus arteriosus. There were no complications neither mortality. The Neonate Intensive Care Unit was important for the evolution of these patients. Our study showed good results achieved by this technique, with low mortality and morbidity and the importance of the Intensive Care Unit for the evolution of these patients.

DESCRIPTORS: Patent ductus arteriosus, surgery, premature neonates. Premature neonates, respiratory insufficiency, surgery. Premature neonates, cardiac insufficiency, surgery. 
Herdy CDC, Abilio FM, Vieira N, Brancaglion B, Castro L S, Oliveira J CP, Pinto C A M, Thomaz J B, Azevedo S L-Correçāo cirúrgica da persistência do canal arterial em crianças de baixo peso e neonatos.

\section{REFERÉNCIAS BIBLIOGRÁFICAS}

1 Barst R J\& Gersony W M . The pharmacological treatment of patent ductus arteriosus. Drugs, 1989; 38: 249-66.

2 Cleveland R J, Nelson R J, Emmanouilides G C - Surgical management of patent ductus arteriosus in infancy. Arch Surg 1969; 99: 516-20.

Edmunds Jr. L H - Operation or indomethacin for premature ductus. Ann Thorac Surg 1978; 26: 586-9.

4 Siegal R E - Galen's experience and observation on pulmonary blood flow and respiration. Am J Cardiol 1962; 10: 738-45.

5 Skoda J - Protokolf des sections-sitaung fur Physiologie und Pathologie. Wbi Z Gis Aertze 1855; $1: 720-2$.

6 Langer C - Zur anatomis der totalen Kreislauef organe. $Z$ Ges Wien Artze 1857; 13: 328-38.

7 Graybriel A, Strieder J W, Boyer W H. An attempt to obliterate the patent ductus arteriosus in a patient with subacute bacterial endocarditis. Am Heart $J$ 1938; 15: $621-4$.

8 Gross RE \& Hubbard J P - Surgical ligation of a patent ductus arteriosus: report of first successfui case. Jama 1939; 112: 729-31.

9 Edwards D K, Wayne M D, Northway W H - Tweive year's experience with bronchopulmonary dysplasia. Pediatries 1977; 59 : 839.46.

10 Friedman W F, Hirschlau M J, Printz M P, Pitlick P L . Pharmacologic closure of ductus arteriosus in pre. mature infants. $N$ Engl $J$ Med 1976; 295: 526-9.

11 Meier M A, Jasbik W, Coutinho J H et al. - Manuseio do canal arterial patente no prematuro com síndrome de angústia respiratória: ligadura ou indometacina? Rev Bras Cir Cardiovasc 1989; 4: 9-20.

12 Cassady G, Crouse D T, Kirklin J W et al. - A randomized controled trial of very early prophylatic ligation of ductus arteriosus in babies who weighed $1000 \mathrm{~g}$ or less at birth. N Engl J Med 1989; 320: 1511.
13 Macruz R \& Snitcowsky R, eds. Cardiologia pediátrica. Sāo Paulo: Sarvier, 1983.

14 Way A L, Pierce Y R, Wolfe R R et al, - ST depression suggesting subendorcadial ischemia in neonates with respiratory distress syndrome and patent ductus arteriosus. J. Pediatr 1979; 95: 609-11.

15 Rudd P, Montanez P, Silverman M - Indomethacin treatment for patent ductus arteriosus in very-low. birth-weight infants: double blind trial. Arch Dis Child $1983 ; 58: 267-70$.

16 Gersony W M, Pecham G J, Ellison R C, Miettinen O S. Nadas A S - Effects of indomethacin in premature infants with patent ductus arteriosus: results of a national collaborative study. J Pediatr 1983; 102: 895-906.

Palder S B, Schwartz M Z, Tyson K R T, Marr C C. Management of patent ductus arteriosus: a comparision of operative versus pharmacological treatment. J Pediatr Surg 1987; 22: 1171-4.

Sharpe G L. Pharmacology of prostaglandin synthetase inhibitors. In: Proceedings of the 75th Ross Conference on Pediatric Research. Palm Beach: 1977.

19 Caidera $R$ \& Badoual $\mathrm{J}$ - Vitamine $E$ et fermeture du canal arteriel du prématuré. Arch Fr Pediatr 1981; 38: 817 .

20 Heymann E, Ohisson A, Shennan A T, Heilbut M, Coceani F- Closure of patent ductus arteriosus after treatment with dexamethasone. Acta Pediatr Scand 1990; 79: $698-700$

21 Brandt B, Marvin W J et al. - Ligation of patent ductus arteriosus in premature infants. Ann Thorac Surg 1981; $32: 167-72$.

22 Coster D D, Gorton M E, Schneider A F et al. - Surgical closure of patent ductus arteriosus in the neonatal care unit. Ann. Thorac Surg 1989; 48: 386-9.

23 Hoffman M, Greve H, Kortman - Operative versehlub des persistierenden ductus arteriosus bei kleinen fruhgeborenen im inkubator. Klin Padiatr 1991: 203: 20-3. 